\title{
The genetics of smoking in individuals with chronic obstructive pulmonary disease
}

\author{
Ma'en Obeidat ${ }^{*}$ (D), Guohai Zhou ${ }^{1}$, Xuan Li ${ }^{1}$, Nadia N. Hansel ${ }^{2}$, Nicholas Rafaels ${ }^{3}$, Rasika Mathias ${ }^{4}$, Ingo Ruczinski ${ }^{5}$, \\ Terri H. Beaty ${ }^{6}$, Kathleen C. Barnes ${ }^{3}$, Peter D. Paré ${ }^{1,7}$ and Don D. Sin ${ }^{1,7}$
}

\begin{abstract}
Background: Smoking is the principal modifiable environmental risk factor for chronic obstructive pulmonary disease (COPD) which affects 300 million people and is the 3rd leading cause of death worldwide. Most of the genetic studies of smoking have relied on self-reported smoking status which is vulnerable to reporting and recall bias. Using data from the Lung Health Study (LHS), we sought to identify genetic variants associated with quantitative smoking and cessation in individuals with mild to moderate COPD.
\end{abstract}

Methods: The LHS is a longitudinal multicenter study of mild-to-moderate COPD subjects who were all smokers at recruitment. We performed genome-wide association studies (GWASs) for salivary cotinine $(n=4024)$, exhaled carbon monoxide (eCO) $(n=2854)$, cigarettes per day (CPD) $(n=2706)$ and smoking cessation at year 5 follow-up $(n=$ 717 quitters and 2175 smokers). The GWAS analyses were adjusted for age, gender, and genetic principal components.

Results: For cotinine levels, SNPs near UGT2B10 gene achieved genome-wide significance (i.e. $P<5 \times 10^{-8}$ ) with top SNP rs10023464, $P=1.27 \times 10^{-11}$. For eCO levels, one significant SNP was identified which mapped to the CHRNA3 gene $\left(r s 12914385, P=2.38 \times 10^{-8}\right)$. A borderline region mapping to $K C N M A 1$ gene was associated with smoking cessation ( $r$ 2207675, $P=5.95 \times 10^{-8}$ ). Of the identified loci, only the CHRNA3/5 locus showed significant associations with lung function but only in heavy smokers. No regions met genome-wide significance for CPD.

Conclusion: The study demonstrates that using objective measures of smoking such as eCO and/or salivary cotinine can more precisely capture the genetic contribution to multiple aspects of smoking behaviour. The KCNMA1 gene association with smoking cessation may represent a potential therapeutic target and warrants further studies.

Trial registration: The Lung Health Study ClinicalTrials.gov Identifier: NCT00000568. Date of registration: October 28, 1999.

Keywords: Cessation, Smoking, GWAS, eCO, Cotinine

\section{Background}

The smoking epidemic is one of the biggest public health challenges in modern history [1]. Tobacco-attributable deaths are expected to rise to more than 10 million globally by 2030 [2-4]. Despite aggressive public health programs aimed at eliminating smoking in the United States (US) and elsewhere, one in 4 adults in the US still use tobacco products and 1 in 5 are daily users. Smoking is the principal modifiable environmental risk factor for chronic

\footnotetext{
* Correspondence: maen.obeidat@hli.ubc.ca

'The University of British Columbia Center for Heart Lung Innovation, St Paul's Hospital, Vancouver, BC, Canada

Full list of author information is available at the end of the article
}

obstructive pulmonary disease (COPD), ischemic heart disease, and lung cancer [5]. COPD, for instance, affects 300 million people and is the 3rd leading cause of death worldwide [6].

Genetic studies of smoking behaviour and smokingrelated illnesses such as COPD and lung cancer have identified strong associations in the chromosome 15q25 region, which contains genes encoding the nicotinic receptor subunits CHRNA3-CHRNA5-CHRNB4 [7-9]. However, most of these studies have relied on selfreported smoking status as either the phenotype of interest or one of the covariates to "adjust" tobacco exposure. Self-report is vulnerable to reporting and recall bias and 
has been shown to consistently underestimate total tobacco exposure $[10,11]$, which may result in residual confounding [12]. One way to mitigate this risk is to use an objective biochemical assay to validate self-reports of tobacco use. The most commonly used assays include exhaled carbon monoxide (eCO) [13] or cotinine in serum, urine or saliva [14]. Because $\mathrm{eCO}$ has a short half-life $(\sim 4 \mathrm{~h})$ it is best suited for short term tobacco exposure while the longer half-life of cotinine $(\sim 16 \mathrm{~h})$ makes it a more robust measure to differentiate active from non-active smokers with a longer duration of abstinence. However, because cotinine is a by-product of nicotine, enzymatic processes involved in nicotine metabolism can affect cotinine levels in smokers. eCO and/or cotinine assays are relatively inexpensive, noninvasive and well-standardized measurements and most importantly they more accurately quantify tobacco exposure in smokers compared with self-report alone $[13,15]$.

To date, very few studies have ascertained the genetic drivers of smoking (and cessation) using validated biochemical assays [16], especially among those with established smoking related diseases such as COPD. Using data from the Lung Health Study, we identified the genetic variants associated with cigarette smoking using validated objective assays in individuals with mild to moderate COPD.

\section{Methods}

\section{The lung health study (LHS)}

The details of the LHS have been published previously $[17,18]$. Briefly, LHS was a multicenter clinical study that evaluated the effects of ipratropium bromide, a short acting antimuscarinic agent (i.e. ipratropium bromide), and smoking cessation on lung function decline in current smokers with mild to moderate COPD. At the time of recruitment all subjects were active smokers between the ages of 35 and 60 years (with a mean age of 48 years) who had smoked at least 10 cigarettes a day within the 30 days prior to initial screening and who demonstrated mild to moderate COPD on spirometry defined by forced expiratory volume in 1 second $\left(\mathrm{FEV}_{1}\right)$ between $55 \%$ and $90 \%$ of predicted, in the presence of a $\mathrm{FEV}_{1} /$ forced vital capacity $(\mathrm{FVC})$ ratio of $<0$. 70 after bronchodilation. The mean $\mathrm{FEV}_{1}$ of the cohort was $75 \%$ predicted and the mean $\mathrm{FEV}_{1} / \mathrm{FVC}$ was $63 \%$ post-bronchodilator.

After enrolment, these patients were randomly assigned to one of 3 groups: (1) usual care (UC), who received no intervention, $n=1964$; (2) an intense antismoking (special) intervention and ipratropium bromide (Atrovent ${ }^{\circ}$, Boehringer Ingelheim Pharmaceuticals) $n=$ 1961 (SIA); or (3) an intense anti-smoking (special) intervention and an inhaled placebo, $n=1962$ (SIP). Ten centers participated in the original study and together they recruited 5887 patients (of whom 37\% were females). Those who were in the SIP or SIA groups received a program that consisted of: 1) a strong recommendation by attending physician for smoking cessation in an one-on-one encounter; 2) a group program led by a health educator that met 12 times over 10 weeks, which taught behavioural modification techniques; and 3) nicotine replacement therapy with nicotine gum (2 mg per piece, Nicorette Gum, Marion Merrell Dow Inc), which was provided at no cost to patients. Those who successfully quit smoking were enrolled in a maintenance program to prevent relapses.

For the first 5 years, the lung function of participants was measured annually. At each face to face visit the subjects' smoking status was determined using a questionnaire, which was validated by salivary cotinine and exhaled carbon monoxide levels as previously described [18]. Participants were classified as smokers if their cotinine levels were greater than $20 \mathrm{ng} / \mathrm{mL}$ or if their exhaled carbon monoxide concentrations were higher than $10 \mathrm{ppm}$. At year 5 of the study, participants were divided into three groups based on smoking history as previously described [19]. Sustained quitters (SQs) were defined as those who gave a history of total abstinence (no month in which the subject smoked even a single cigarette per day) and had eCO readings below $10 \mathrm{ppm}$ at each annual follow-up visit over 5 years. Continuing smokers (CSs) were those who reported smoking at all scheduled follow-up visits. Intermittent quitters (IQs) were current smokers at some but not all of their visits. Given the ambiguity of the IQ group $(n=1210)$ in terms of cigarette smoking, they were excluded from the smoking cessation genome wide association study (GWAS).

\section{Genotyping}

At year 5 of LHS, venipuncture was carried out on 5413 LHS participants who were alive and eligible at this visit. Blood samples were taken when participants were stable and free of exacerbations for at least 4 weeks and were separated into buffy coat and serum [20]. DNA was extracted from the buffy coat samples of 4251 European Americans in LHS and SNP genotyping was performed. The details of genotyping and quality control have been previously described [21]. Briefly, samples were genotyped using the Illumina Human660WQuad v.1_A BeadChip. Overall, 98. $4 \%$ of samples $(n=4181)$ passed initial quality control standards and genotypes were available for 559,766 SNPs. An additional 133 samples were removed because they failed subsequent quality control, which resulted in a final sample of $n=4048$ for the present analysis. Imputation was undertaken with the Michigan Imputation Server [22] using the Haplotype Reference Consortium (HRC) [23] panel. Variants were excluded if the imputation $r^{2}$ was $<0.5$ and if the minor allele frequency was $<1 \%$. 


\section{Measurements of expired carbon monoxide (eCO) and cotinine}

The details of eCO and cotinine measures have been previously described [24]. To conduct the cotinine assay, LHS participants were asked to deposit at least $1 \mathrm{ml}$ of saliva in a plastic vial, which was then frozen and sent in a batch to the American Health Foundation laboratory in Valhalla, NY. One sample was taken from each participant and a single cotinine assay measurement was performed. The cotinine assessment was conducted using the radioimmunoassay technique of Langone et al. by personnel who were blinded to the smoking status of the participants [24]. In LHS, the sensitivity and specificity of using a salivary cotinine cutoff of $20 \mathrm{ng} / \mathrm{ml}$ compared to self-report was $99 \%$ and $92 \%$, respectively. Studies have reported a technical coefficient of variation (CV) value of $5 \%$ for salivary cotinine assay [25].

Carbon monoxide in expired air was measured using either of two instruments: the MiniCO (Catalyst Research) or the EC50 (Vitalograph). The eCO measurement procedure involved two attempts. If the two values were not within $4 \mathrm{ppm}$, the measurements were repeated. The result was the average of the two readings rounded to the nearest integer [24].

\section{Genome-wide association analyses (GWASs)}

Given that eCO, cotinine and cigarettes per day (CPD) distributions were skewed, the values were transformed into normally distributed $\mathrm{Z}$ scores using the $\mathrm{R}$ function 'rntransform' [26] (Additional file 1: Figure S1). We performed GWAS for three transformed phenotypes: eCO, salivary cotinine and CPD using SNPTEST [27] assuming an additive genetic model and adjusting for age, gender, and the first 5 genetic principal components (PCs). Since cotinine levels were measured in all subjects at baseline visit, we performed the GWAS for cotinine baseline levels to make available the largest sample size $(n=4024$ and missing data rate $=1.9 \%$ ). The $\mathrm{eCO}$ and $\mathrm{CPD}$ values were only measured in smokers at subsequent visits so we used the values measured at year 1 follow up to make available the largest sample size $(n=2706$ and missing data rate $=8$. $1 \%$ for eCO; $n=2854$ and missing data rate $=3.12 \%$ for CPD). Significant SNPs were defined as the sentinel SNPs meeting genome-wide significance $\left(P<5 \times 10^{-8}\right)$.

\section{Evaluation of previously published variants which relate to smoking cessation}

We evaluated previously published hits from two studies for smoking cessation. The first study is the Tobacco and Genetic Consortium (TAG) meta-analysis GWAS of smoking behaviour [28]. The TAG study included 41,278 former and current smokers and the top 15 associated SNPs for self-reported smoking cessation were followed up in 64,924 independent individuals from the European
Network of Genetic and Genomic Epidemiology (ENGAGE) and the Oxford-GlaxoSmithKline (Ox-GSK) consortia. This meta-analysis identified only one significant SNP as being associated with smoking cessation; rs3025343 $\left(P=1.8 \times 10^{-8}\right)$, which was located on chromosome 9, near the dopamine beta-hydroxylase $(\mathrm{DBH})$ gene.

The second study by Siedlinski et al. reported GWAS results for self reported phenotypes of smoking including lifetime average and current CPD, age at smoking initiation, and smoking cessation in 3441 patients with COPD [29]. In total, the meta-analysis included 1164 current smokers and 1907 former smokers (all using self report of yes/no answers); none of the SNPs had showed a statistically significant association with smoking cessation. In the present study, we interrogated the 9 SNPs associated with smoking cessation reported in Siedlinski et al. study.

\section{Association of smoking related SNPs with extremes of lung function}

To determine whether any of the smoking related SNPs discovered in the present study also had an impact on smoking-related physiological outcomes such as lung function, we tested SNPs identified in this study for association with lung function in never and, separately, in heavy smokers. The UK Biobank Lung Exome Variant Evaluation (UK BiLEVE) evaluated the genetic determinants related to low (mean of $65.6 \%$ predicted, average (mean of $90.6 \%$ predicted), or high (mean of $118 \%$ predicted) forced expiratory volume in 1 second $\left(\mathrm{FEV}_{1}\right)$ in heavy smokers (mean 35 pack-years) and separately in never smokers. The study included 10,002 individuals with low $\mathrm{FEV}_{1}, 10,000$ with average $\mathrm{FEV}_{1}$, and 5002 with high $\mathrm{FEV}_{1}$ from each of the heavy smoker and never smoker groups. Genome-wide genotyping was performed using a custom Affymetrix Axiom array (UK BiLEVE array; Santa Clara, CA, USA). After quality control nongenotyped variants were imputed using a combined 1000G Phase 1 and UK10K Project [30] reference panel.

\section{Gene drug interactions}

To uncover the potential biological relevance of the smoking cessation GWAS hits, we used two databases to search for potential gene drug interactions: the DGIdb [31] http://www.dgidb.org/ and the DRUGBANK database [32] https://www.drugbank.ca/ .

\section{Results}

\section{Descriptive demographics of LHS participants}

The overall LHS design and the sample size for each of the GWASs are shown in Fig. 1, and the demographics and quantitative smoking values are shown in Table 1. Among smokers at year 1 of LHS, there was a strong 


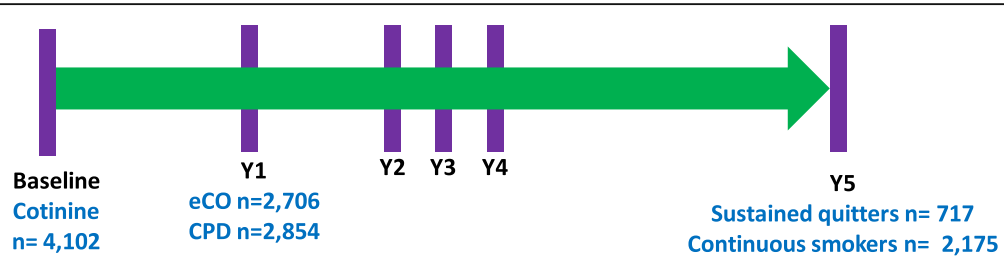

Fig. 1 Overall LHS smoking GWAS study design. eCO: exhaled carbon monoxide. CPD: Cigarettes per day. Y1: year 1. eCO was measured in those reporting current smoking

correlation between eCO, cotinine, and CPD values with the correlation between $\mathrm{eCO}$ and $\mathrm{CPD}$ being the strongest $\left(r=0.5, P=1 \times 10^{-170}\right.$, Additional file 1: Figure S2).

The levels of quantitative smoking biomarkers such as cotinine were inversely related to cross sectional lung function measures at years 1-5 (Additional file 1: Figure S3). This relationship was significant $(P<0.05)$ for years $1-4$ and borderline significant in year $5(P=0.08)$. Furthermore, cotinine levels at year 5 showed negative correlation with FEV1 decline between years 1 and $5\left(P=3 \times 10^{-11}\right.$, Additional file 1: Figure S4).

\section{Genome-wide association results for cotinine levels}

In the four GWAS analyses, we included 7,807,992 variants with $\mathrm{MAF}>1 \%$ and imputation quality $>0.5$. The GWAS of cotinine levels at baseline included 4024 individuals. Quantile-quantile (QQ) plots are presented in Additional file 1: Figure S5, which showed a sharp deviation from the expected distribution for low $p$-values indicating strong signals. The genomic inflation factor $(\lambda)$ was 0.996 , suggesting no systematic deviation in the association statistics due to factors such as population structure.

A total of 250 SNPs in the $4 \mathrm{q} 13.2$ region containing the UGT2B10 gene achieved genome-wide significance $\left(P<5 \times 10^{-8}\right)$. A Manhattan plot is shown in Fig. 2 and the region plots are shown in Fig. 3. The most significantly associated SNP for cotinine levels was an intergenic SNP on chromosome $4($ rs10023464) $(P=1$. $\left.27 \times 10^{-11}\right)$. Another interesting signal was observed on the 15q25.1 region with an intronic SNP rs9788721 $(P=$ $3.49 \times 10^{-7}$ ) in the HYKK gene near CHRNA3/5 genes, though this latter association did not reach genomewide significance. All SNPs meeting or approaching genome-wide significance for smoking phenotypes are presented in Table 2.

The association of the two cotinine-associated loci (UGT2B10 and CHRNA3/5) with cotinine levels at subsequent years showed that the strength of the association decays during subsequent follow up visits i.e. the $P$ values increase yet they maintain the same direction of effect (Additional file 1: Table S1). This decrease in significance is directly proportional to the the decrease in sample sizes available for the analysis at follow up visits with a missing rate ranging from $8 \%$ in year 1 to $51 \%$ missing subjects at year 5 (Additional file 1: Table S2).

\section{Genome-wide association results for exhaled carbon monoxide (eCO) levels}

The GWAS of eCO levels at year 1 included 2706 smokers (Fig. 2 and Additional file 1: Figure S5). The genomic inflation factor $(\lambda)$ was 1.00 . Only one region; the $15 \mathrm{q} 25.1$ region met genome wide significance with one SNP; intronic SNP (rs12914385) mapping to the CHRNA3 gene $\left(P=2.38 \times 10^{-8}\right)$ (Fig. 3 region plots). Two regions approached genome wide significance for eCO. The first was the 3q22.3 region with intronic SNP rs546764 $\left(P=7.76 \times 10^{-8}\right)$ mapping to the CEP70 gene.

Table 1 Demographics of study subjects. Gender male in n (\% of column totals), other variables in mean \pm SD. CPD: cigarettes per day. eCO exhaled carbon monoxide. * Age at assessment

\begin{tabular}{|c|c|c|c|c|c|c|}
\hline & \multirow{2}{*}{$\begin{array}{l}\text { Baseline } \\
\text { Smokers }\end{array}$} & \multicolumn{2}{|l|}{ Year 1} & \multicolumn{3}{|l|}{ Year 5} \\
\hline & & Smokers & Quitters & Continuous smokers & Sustained quitters & Intermittent quitters \\
\hline$\overline{N(\%)}$ & 4102 & 2946 & 1156 & 2175 & 717 & 1210 \\
\hline Age $^{*}$ (years) & $48.6 \pm 6.7$ & $49.4 \pm 7$ & $50.0 \pm 6.7$ & $53.3 \pm 6.7$ & $54.2 \pm 6.6$ & $53.7 \pm 6.7$ \\
\hline Gender (male) & $2853(63 \%)$ & $1837(62 \%)$ & $746(65 \%)$ & $1366(63 \%)$ & $481(67 \%)$ & 736 (61\%) \\
\hline $\mathrm{BMI}\left(\mathrm{kg} / \mathrm{m}^{2}\right)$ & $25.5 \pm 3.8$ & $25.7 \pm 3.9$ & $27.3 \pm 4.0$ & $26.0 \pm 4.2$ & $28.4 \pm 4.3$ & $27.5 \pm 4.2$ \\
\hline FEV1\% predicted & $78.6 \pm 9.0$ & $78.0 \pm 9.8$ & $81.8 \pm 9.5$ & $72.8 \pm 12.0$ & $80.3 \pm 10.9$ & $77.2 \pm 11.5$ \\
\hline eCO (ppm) & $32.4 \pm 16.1$ & $24.8 \pm 13.2$ & $4.8 \pm 2.5$ & $25.4 \pm 13.8$ & $4.0 \pm 2.4$ & $9.6 \pm 11.1$ \\
\hline Cotinine (ng/ml) & $361.4 \pm 199.3$ & $302.7 \pm 146.3$ & $92.2 \pm 158.7$ & $343.1 \pm 195.3$ & $27.8 \pm 132.4$ & $117.5 \pm 229.6$ \\
\hline CPD cigarettes/day & $21.9 \pm 14.5$ & $21.9 \pm 14.5$ & $0 \pm 0$ & $23.0 \pm 12.9$ & $0 \pm 0$ & $5.3 \pm 10.2$ \\
\hline
\end{tabular}




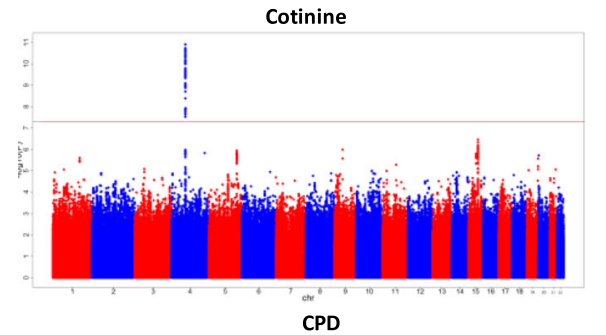

CPD

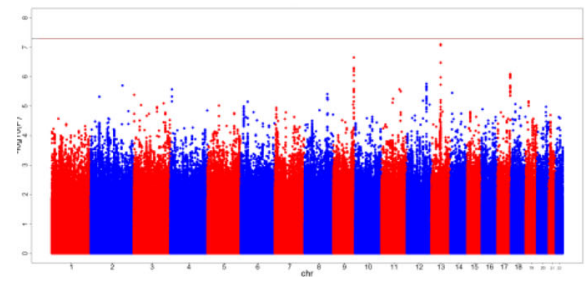

eco

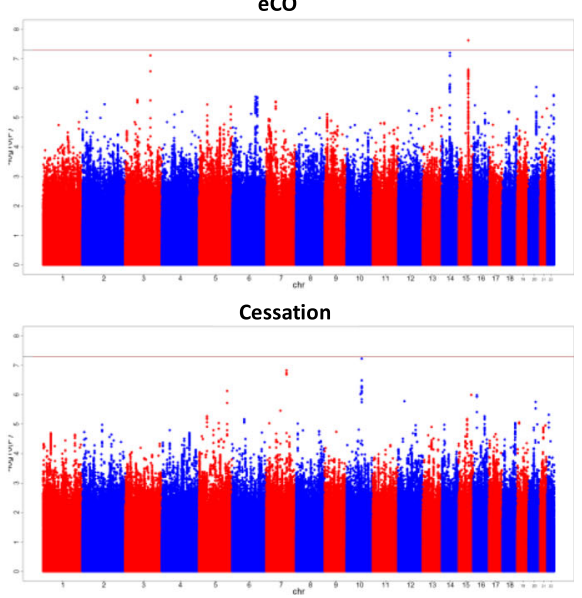

Fig. 2 Manhattan plots of smoking GWASs in Lung Health Study. The plots show the $P$ values (-log10 scale) on the Y axes and the SNP positions across 22 autosomal chromosomes on the $X$ axes. The horizontal red line represents the genome-wide cut-off of $5 \times 10^{-08}$

The second region was the $14 \mathrm{q} 23.1$ region with an intergenic SNP rs140706189 $\left(P=6.31 \times 10^{-8}\right)$ near the SIX1/4/6 and MNAT1 genes (Table 2).

\section{Genome-wide association results for cigarettes per day (CPD)}

The GWAS for CPD included a total of 2854 smokers at year 1 (Fig. 2 and Additional file 1: Figure S5). The genomic inflation factor $(\lambda)$ was 1.00 . No regions met genome-wide significance. Two regions, however, approached this level of significance. These included the 13q21.32 region with intronic SNP rs9599114 mapping to $\mathrm{PCDH} 9$ gene $\left(P=7.94 \times 10^{-8}\right)$, and the $9 \mathrm{q} 34.13$ region with intergenic SNP rs1412076 $\left(P=2.24 \times 10^{-8}\right)$ near NTNG2 (Table 2).

\section{Genome-wide association results for smoking cessation}

At year 5 of LHS, there were 717 sustained quitters and 2175 continuous smokers (quitters vs. smokers case control GWAS). No loci met genome-wide significance but two loci very closely approached this level of significance (Figs. 2 and 3). The strongest association was intronic SNP rs207675, which mapped to the KCNMA1 gene on 10q22.3 $\left(P=5.95 \times 10^{-8}\right)$. The second loci included the intronic SNP $\operatorname{rs} 212420 \quad\left(P=1.50 \times 10^{-8}\right)$ near the ATXN7L1 gene on 7q22.3.

\section{Evaluation of previously associated SNPs for smoking behaviours}

Previous reports have identified genetic loci (CHRNB3/ A6 region on chromosome 8 and the CYP2A6 region on chromosome 19) that were significantly associated with CPD [33] . In the present study, both of these SNPs showed no significant association with CPD $(P>0.05)$. Additionally, we evaluated 15 SNPs that were nominally related to smoking cessation in two previous publications [28] and a number of other SNPs that were nominally associated with smoking cessation among COPD subjects [29].

Additional file 1: Table S3 shows the results of the look-up. One SNP; rs4362358 near the CHRNA3/5 genes that was related to cessation in the TAG consortium [28] was associated with eCO $(P=0.03)$ and cotinine $(P=0.002)$ in our study. Another cessation SNP in the TAG consortium; rs17178639 in SLC25A21 gene was associated with eCO $(P=0.007)$ in the present study. The two SNPs were associated with reduced cessation in the TAG consortium and were also associated with higher eCO and cotinine levels in our study. Of the cessation SNPs in COPD patients from the study of Siedlinski et al. [29], two near the IPMKP1 gene were nominally associated with cessation in our study: rs9506942, and rs9552733 with $P=0.005$ and $P=0.004$, respectively and with the same direction of effect.

We tested SNPs identified in our study for associations with smoking phenotypes in the TAG consortium (CPD, cessation, age of onset and ever vs. never phenotypes). Only CHRNA3/5 SNPs (for eCO and cotinine) were significant in the TAG data for both CPD and for cessation (Additional file 1: Table S4).

\section{Associations with lung function}

We tested the SNPs identified in this study for associations with extremes of lung function: high vs. low FEV1 in heavy smokers and separately for never smokers in a large study from the UK Biobank [34]. Only the CHRNA3/5 region variants showed associations with lung function and only in the heavy smokers group (not in the never smokers). The results for lung function are shown in Additional file 1: Table S5. 

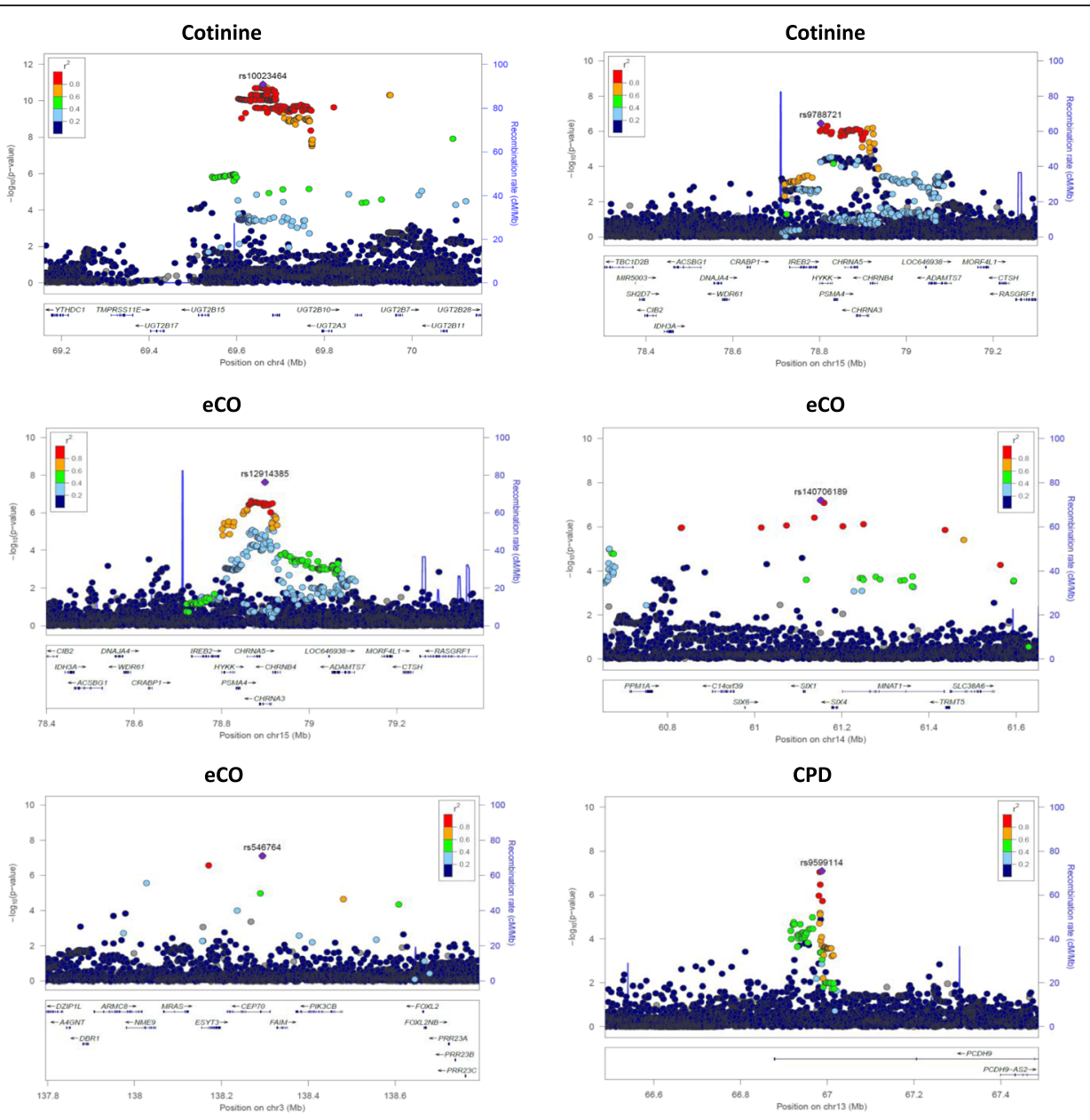

CPD
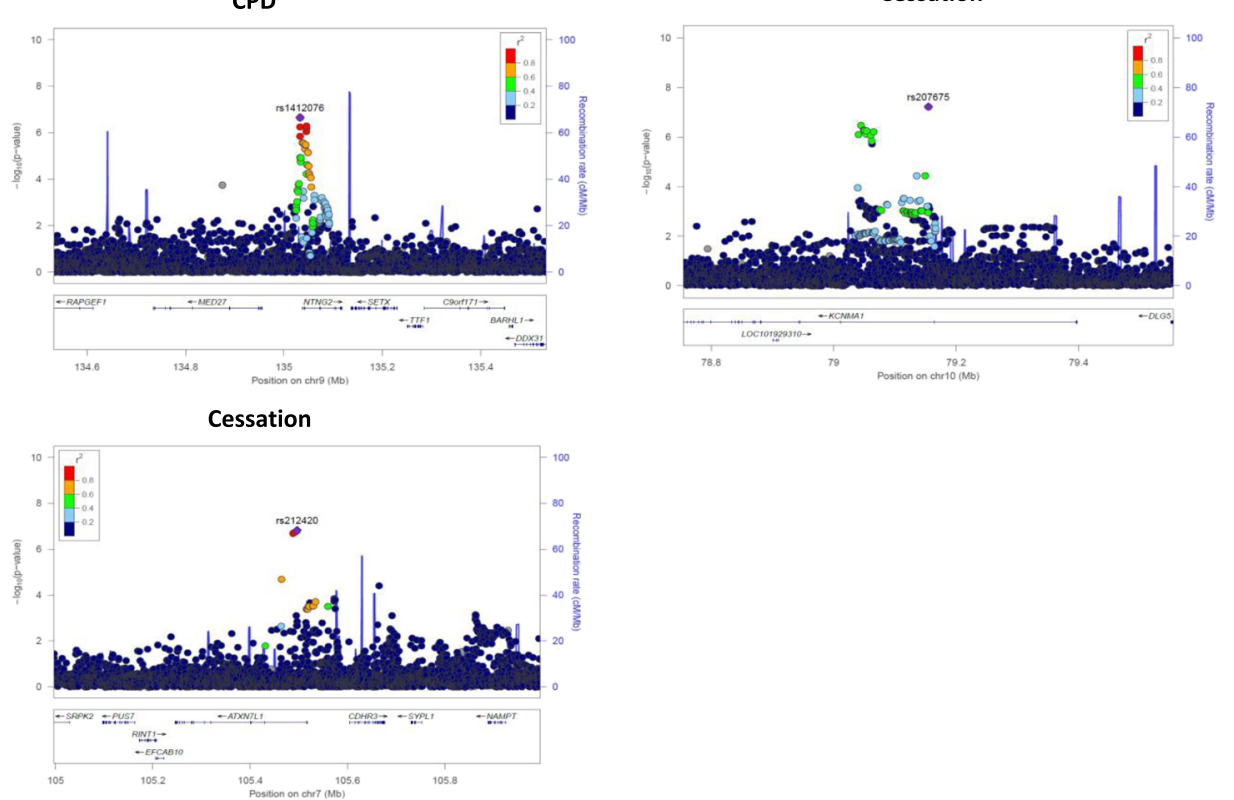

Fig. 3 Region plots of the smoking associated loci. The $Y$ axis represent the $P$ values in the (-log10 scale) and the $X$ axis is the genomic position. Gene names and their corresponding coordinates are shown below. The sentinel SNP is shown as a purple diamond and the color coding of SNPs reflects the degree of linkage disequilibrium (LD) with the sentinel SNP using 1000G reference 
Table 2 Genetic loci associated with smoking behaviour in the Lung Health Study

\begin{tabular}{|c|c|c|c|c|c|c|c|c|c|c|c|}
\hline Phenotype & SNP & $\mathrm{Chr}$ & Gene(s) & Position (hg19) & Alleles (REF/ALT) & MAF & Imputation r2 & beta & SE & $P$ value & $\%$ Variance \\
\hline Cotinine & rs10023464 & 4 & UGT2B10 & $69,659,738$ & $\mathrm{C} / \mathrm{T}$ & $9.8 \%$ & 0.995 & 0.25 & 0.04 & $1.27 \mathrm{e}-11$ & $1.1 \%$ \\
\hline Cotinine & rs9788721 & 15 & CHRNA3/5 & $78,802,869$ & $\mathrm{C} / \mathrm{T}$ & $39.5 \%$ & 0.989 & -0.12 & 0.02 & $3.49 e-7$ & $0.6 \%$ \\
\hline $\mathrm{eCO}$ & rs546764 & 3 & CEP70 & $138,294,336$ & $\mathrm{~T} / \mathrm{G}$ & $2.8 \%$ & 0.844 & 0.39 & 0.07 & $7.76 \mathrm{e}-8$ & $0.9 \%$ \\
\hline eCO & rs140706189 & 14 & SIX1/4/6, MNAT1 & $61,151,425$ & $\mathrm{~T} / \mathrm{G}$ & $1.7 \%$ & 0.827 & -0.49 & 0.09 & $6.31 \mathrm{e}-8$ & $0.9 \%$ \\
\hline eCO & rs12914385 & 15 & CHRNA3/5 & $78,898,723$ & $\mathrm{C} / \mathrm{T}$ & $43 \%$ & 1.000 & 0.12 & 0.02 & $2.38 \mathrm{e}-8$ & $1.2 \%$ \\
\hline CPD & rs1412076 & 9 & NTNG2/ SETX & $135,032,890$ & $\mathrm{~A} / \mathrm{G}$ & $37.6 \%$ & 0.999 & 0.10 & 0.02 & $2.24 \mathrm{e}-7$ & $0.8 \%$ \\
\hline CPD & rs9599114 & 13 & $\mathrm{PCDH} 9$ & $66,987,131$ & $\mathrm{~T} / \mathrm{C}$ & $41.7 \%$ & 0.957 & 0.11 & 0.02 & $7.94 \mathrm{e}-8$ & $0.8 \%$ \\
\hline Cessation & rs212420 & 7 & ATXN7L1/ CDHR3 & $105,496,412$ & $C / G$ & $6.7 \%$ & 0.992 & -0.60 & 0.11 & $1.50 \mathrm{e}-7$ & $\mathrm{n} / \mathrm{a}$ \\
\hline Cessation & rs207675 & 10 & KCNMA1 & $79,154,537$ & $\mathrm{~T} / \mathrm{C}$ & $33.6 \%$ & $0 . .988$ & -0.35 & 0.06 & $5.95 e-8$ & $\mathrm{n} / \mathrm{a}$ \\
\hline
\end{tabular}

UGT2B10 UDP glucuronosyltransferase family 2 member B10, CHRNA3 cholinergic receptor nicotinic alpha 3 subunit, CHRNA5 cholinergic receptor nicotinic alpha 5 subunit, CEP70 centrosomal protein 70, SIX1 SIX homeobox 1, MNAT1 MNAT1, CDK activating kinase assembly factor, NTNG2 Nitrin G2, SETX senataxin, PCDH9 protocadherin 9, ATXN7L1 ataxin 7 like 1, CDHR3 cadherin related family member 3, KCNMA1 potassium calcium-activated channel subfamily M alpha $1,{ }^{*}$ Refers to odds ratio and $95 \%$ confidence intervals $(\mathrm{Cl})$

\section{Discussion}

Smoking places a huge burden on individuals and health care systems. Smoking behaviours, and consequently the risk of smoking related illnesses are at least partially genetically determined $[35,36]$. The majority of previous studies on the genetics of smoking behaviour have relied on self-report, which is affected by recall bias and more importantly under-reporting bias, which may lead to an inaccurate assessment of smoking exposure. Indeed, several groups have shown that in approximately 25 to $50 \%$ of self-reported quitters, objective assays could not validate the reported smoking status [37] . It is thus crucial to accurately phenotype smoking status to properly understand the molecular mechanisms underlying nicotine addiction, metabolism, and smoking cessation.

In the current study of COPD subjects, we performed GWAS for the phenotypes of self-reported cigarettes per day (CPD) and two biochemical biomarkers of smoking: $\mathrm{eCO}$ and salivary cotinine. Additionally, we evaluated genetic determinants of biochemically validated smoking cessation over 5 years. Using this approach, we identified genome-wide significant loci associated with salivary cotinine on 15q.25.1 (CHRNA3/5 genes) and 4q13.2 (UTG2B10) gene). For eCO, only the 15q.25.1 locus reached genome-wide significance. The smoking cessation GWAS revealed a borderline signal in the KCNMA1 gene on 10q22.3. Finally, of all the loci identified in the current study, only the CHRNA3/5 locus showed a significant association with lung function in heavy smokers (but not in never smokers) from the general population.

Genetic determinants of smoking can be related to smoking intensity (addiction), metabolism or both. The metabolism of nicotine involves several enzymatic pathways. Approximately $10 \%$ of nicotine is excreted unchanged in the urine. The majority of nicotine $(\sim 80 \%)$ is converted to cotinine in two steps: initial metabolism, which is mediated by the cytochrome P450, family 2 , subfamily A, poly-peptide 6 (CYP2A6) enzyme, followed by conjugation by aldehyde oxidase [38]. After these two steps cotinine is further metabolised by CYP2A6 to 3hydroxycotinine. Oxidation and glucuronidation processes account for the remaining $10 \%$ of the metabolic process [38]. We found significant association of salivary cotinine with SNPs in UDP glucuronosyltransferase family 2 member B10 (UGT2B10), which catalyses both nicotine and cotinine glucuronidation in smokers.

Previous GWAS for cotinine in urine, plasma or serum have all identified the UGT2B10 region [16, 39] with stronger associations reported for urinary cotinine levels. In the current study, we replicated the association signal for SNPs in the UGT2B10 region for association with salivary cotinine. Smokers are thought to self-titrate their nicotine to meet their physiological need [40] (i.e. high metabolizers are likely to smoke more). If this hypothesis were true, then we would expect variations in the rates of metabolism (and hence consumption) to be associated with smoking related diseases/phenotypes. However, we failed to find any significant associations between SNPs in the UGT2B10 gene region with impaired lung function in smokers. We may not have sufficient power to detect a subtle effects of this locus; alternatively the two mechanisms (metabolism and consumption) may not be directly linked as previously suggested [34]. The association results of the cotinine and eCO-associated SNPs with CPD in the same individuals are shown in Additional file 1: Table S6. The results indeed show that the UGT2B10 variant is not associated with CPD $(P=0.28)$, arguing against the notion that variation in metabolism of nicotine may affects smoking behaviour.

Perhaps the most widely studied and reported region for smoking is CHRNA3/5 on 15q25. The associations of this region in the current study are with biochemical biomarkers of smoking (eCO and cotinine). In the present 
study, the CHRNA3/5 variants were associated with salivary cotinine as well as eCO levels. Importantly, these variants were also significantly related to CPD $(P<0.05)$, suggesting this genetic region modulates cigarette consumption (Additional file 1: Table S6). However, this locus was not associated with smoking cessation in LHS participants, suggesting that other factors are involved in quitting. Other previously reported loci in CYP2A6 and CHRNB3/CHRNA6 genes could not be replicated in our study. However, as previously noted $[35,36]$, the strength of the relationship between these loci and smoking is relatively modest and may require much larger sample sizes to be detected.

We identified a suggestive signal $\left(P=5.95 \times 10^{-8}\right)$ for smoking cessation in the potassium calcium-activated channel subfamily $\mathrm{M}$ alpha 1 (KCNMA1) gene which is important for the control of smooth muscle tone and neuronal excitability [41, 42]. The association between the KCNMA1 variant with cessation in our study could not be replicated in the cessation GWAS from the TAG consortium [28]. This could be due to the fact that our study used a biochemically verified smoking status; whereas the previous studied relied only on self-report. Our data are in keeping with a previously published study. A GWAS in Australian and Dutch populations identified SNPs in the KCNMA1 gene, which were significantly associated with nicotine dependence (rs592676, $p=8.91 \times 10^{-6}$ ). In our study, the same SNP was strongly associated with cessation $\left(P=5.6 \times 10^{-7}\right)$. Interestingly, a drug repositioning study that integrated disease and drug expression profiles identified KCNMA1 as a potential molecular target for lobeline: a natural alkaloid that has been used as a smoking cessation aid [43] as well as for amphetamine and cocaine addictions [44] KCNMA1 is a target for the FDA approved drug, chlorzoxazone, which is a centrally acting muscle relaxant. Chlorzoxazone acts as an activator of a calcium-activated potassium channel [45] and is commonly used as a probe drug to phenotype CYP2E1 activity and its metabolism is strongly accelerated by cigarette smoking [46]. Another study proposed chlorzoxazone as a potential treatment for alcohol addiction [47]. At the gene expression level, NHBE cells exposed to nicotine-containing e-cigarette vapour demonstrate decreased expression of KCNMA1 [48], while in human lung tissue smokers have significantly higher expression compared to never smokers ( 1.5 fold change, $P=3.11 \times 10^{-08}$ ) [49]. Finally, a genome-wide study identified differential hydroxymethylation of potassium channel genes, including KCNMA1, in the nucleus accumbens in methamphetamine addiction and abstinence [50]. Taken together, these data suggest the KCNMA1 association with smoking cessation is biologically plausible with the potential for drug repurposing.
This study has several limitations. The sample size may have been too small to detect novel loci for smoking biomarkers or cessation. On the other hand, the use of precise biochemical phenotypes on the other hand likely improved the specificity of the smoking cessation signal. Furthermore, and in line with most published GWASs, the proportion of variance explained by the identified variants is small.

\section{Conclusion}

In conclusion, we identified genetic loci associated with $\mathrm{eCO}$ and cotinine in COPD patients. Our study strongly support the need to use objective measures of smoking to capture the genetic contribution to smoking in these studies. The KCNMA1 region association with smoking cessation represents a potential target for drug discovery and repurposing which warrants further studies.

\section{Additional file}

Additional file 1: Supplementary Figures and Tables. (DOCX 1183 kb)

\section{Acknowledgements}

M. Obeidat is a Fellow of the Parker B. Francis Foundation. He is also a recipient of British Columbia Lung Association Research Grant. D. Sin holds a Tier 1

Canada Research Chair in COPD.

\section{Funding}

This study was funded by the Canadian Respiratory Research Network (CRRN) and Genome Canada: Genome British Columbia. CRRN is supported by grants from the Canadian Institutes of Health Research (CIHR) - Institute of Circulatory and Respiratory Health, Canadian Lung Association/Canadian Thoracic Society, British Columbia Lung Association, and Industry Partners Boehringer Ingelheim Canada Ltd., AstraZeneca Canada Inc. and Novartis Canada Ltd. Funding for training of post-doctoral students and new investigators within the network was supported by the above funding sponsors and as well by GlaxoSmithKline Inc. The funders had no role in the study design, data collection and analysis or preparation of the manuscript.

\section{Availability of data and materials}

The genotype data for the Lung Health Study is available on National Center for Biotechnology Information (NCBI) database of genotypes and phenotypes (dbGaP) under phs000335.v3.p2.

\section{Authors' contributions \\ Conceived and designed the study: MO, PDP, DDS. Lung Health Study data analysis and genotyping: NNH, NR, RM, IR, THB, KCB. Statistical analyses: GZ, MO, XL. Wrote the manuscript: MO, PDP, DDS. Discussed results and implications and commented on the manuscript at all stages: all co-authors. All authors read and approved the final manuscript.}

Ethics approval and consent to participate

Work undertaken in this manuscript was approved by the University of British Columbia Institutional Review Board, certificate numberH16-01201.

\section{Consent for publication}

Not applicable.

\section{Competing interests}

Dr. Sin has received research funding from AstraZeneca (AZ), Merck, Boehringer Ingelheim (Bl) and Novartis and has received honorarium for speaking engagements from AZ, Novartis, Regeneron and Sanofi-Aventis. 


\section{Publisher's Note}

Springer Nature remains neutral with regard to jurisdictional claims in published maps and institutional affiliations.

\begin{abstract}
Author details
${ }^{1}$ The University of British Columbia Center for Heart Lung Innovation, St Paul's Hospital, Vancouver, BC, Canada. ${ }^{2}$ Pulmonary and Critical Care Medicine, School of Medicine, Johns Hopkins University, Baltimore, MD, USA. ${ }^{3}$ Division of Biomedical Informatics and Personalized Medicine, Department of Medicine, University of Colorado School of Medicine, Anschutz Medical Campus, Aurora, CO, USA. ${ }^{4}$ Division of Genetic Epidemiology, School of Medicine, Johns Hopkins University, Baltimore, MD, USA. ${ }^{5}$ Department of Biostatistics, Bloomberg School of Public Health, Johns Hopkins University, Baltimore, MD, USA. ${ }^{6}$ Department of Epidemiology, Bloomberg School of Public Health, Johns Hopkins University, Baltimore, MD, USA. 'Respiratory Division, Department of Medicine, University of British Columbia, Vancouver, BC, Canada.
\end{abstract}

\section{Received: 16 January 2018 Accepted: 27 March 2018}

\section{Published online: 10 April 2018}

\section{References}

1. WHO. Tobacco fact sheet: World Health Organization; 2015. http://www wpro.who.int/mediacentre/factsheets/fs_201203_tobacco/en/.

2. Mortality from smoking in developed countries 1950-2020 [http://www.ctsu.ox.ac.uk/tobacco].

3. Jha P, Peto R. Global effects of smoking, of quitting, and of taxing tobacco. N Engl J Med. 2014;370:60-8.

4. Jha P. Avoidable global cancer deaths and total deaths from smoking. Nat Rev Cancer. 2009:9:655-64.

5. Doll R, Peto R. Mortality in relation to smoking: 20 years' observations on male British doctors. Br Med J. 1976;2:1525-36

6. Lozano R, Naghavi M, Foreman K, Lim S, Shibuya K, Aboyans V, Abraham J, Adair T, Aggarwal R, Ahn SY, et al. Global and regional mortality from 235 causes of death for 20 age groups in 1990 and 2010: a systematic analysis for the global burden of disease study. Lancet. 2010;380:2095-128.

7. Thorgeirsson TE. A variant associated with nicotine dependence, lung cancer and peripheral arterial disease. Nature. 2008;452:638-42.

8. Obeidat ME, Hao K, Bossé Y, Nickle DC, Nie Y, Postma DS, Laviolette $M$ Sandford AJ, Daley DD, Hogg JC, et al. Molecular mechanisms underlying variations in lung function: a systems genetics analysis. Lancet Respir Med. 2015;3:782-95.

9. Hobbs BD, de Jong K, Shrine NRG, Wyss AB, London SJ, Cho M. A genomewide association study of chronic obstructive pulmonary disease identifies 22 risk loci. Nat Genet. 2017;49:426-32.

10. Stelmach R, Fernandes FLA, Carvalho-Pinto RM, Athanazio RA, Rached SZ, Prado GF, Cukier A. Comparison between objective measures of smoking and self-reported smoking status in patients with asthma or COPD: are our patients telling us the truth? J Bras Pneumol. 2015;41:124-32.

11. Fendrich M, Mackesy-Amiti ME, Johnson TP, Hubbell A, Wislar JS. Tobaccoreporting validity in an epidemiological drug-use survey. Addict Behav. 2005;30:175-81.

12. Obeidat ME, Ding X, Fishbane N, Hollander Z, Ng RT, McManus B, Tebbutt SJ, Miller BE, Rennard S, Paré PD, Sin DD. The effect of different case definitions of current smoking on the discovery of smoking-related blood gene expression signatures in chronic obstructive pulmonary disease. Nicotine Tob Res. 2016;18:1903-9.

13. Jarvis MJ, Russell MA, Saloojee Y. Expired air carbon monoxide: a simple breath test of tobacco smoke intake. Br Med J. 1980;281:484-5.

14. Jatlow P, Toll BA, Leary V, Krishnan-Sarin S, O'Malley SS. Comparison of expired carbon monoxide and plasma cotinine as markers of cigarette abstinence. Drug Alcohol Depend. 2008;98:203-9.

15. Sato S, Nishimura K, Koyama H, Tsukino M, Oga T, Hajiro T, Mishima M. Optimal cutoff level of breath carbon monoxide for assessing smoking status in patients with asthma and COPD. Chest. 2003;124:1749-54.

16. Ware JJ, Chen X, Vink J, Loukola A, Minica C, Pool R, Milaneschi Y, Mangino M, Menni C, Chen J, et al. Genome-wide meta-analysis of cotinine levels in cigarette smokers identifies locus at 4q13.2. Sci Rep. 2016;6:20092.

17. Kanner RE, Connett JE, Williams DE, Buist AS. Effects of randomized assignment to a smoking cessation intervention and changes in smoking habits on respiratory symptoms in smokers with early chronic obstructive pulmonary disease: the lung health study. Am J Med. 1999;106:410-6.

18. Anthonisen NR, Connett JE, Kiley JP, Altose MD, Bailey WC, Buist AS, Conway WA Jr, Enright PL, Kanner RE, O'Hara P, et al. Effects of smoking intervention and the use of an inhaled anticholinergic bronchodilator on the rate of decline of FEV1. The lung health study. JAMA. 1994;272:1497-505.

19. Nicholas RA, John EC, Robert PM. Smoking and lung function of lung health study participants after 11 years. Am J Respir Crit Care Med. 2002;166:675-9.

20. Man SF, Connett JE, Anthonisen NR, Wise RA, Tashkin DP, Sin DD. C-reactive protein and mortality in mild to moderate chronic obstructive pulmonary disease. Thorax. 2006;61:849-53.

21. Hansel NN, Ruczinski I, Rafaels N, Sin DD, Daley D, Malinina A, Huang L, Sandford A, Murray T, Kim Y, et al. Genome-wide study identifies two loci associated with lung function decline in mild to moderate COPD. Hum Genet. 2013;132:79-90.

22. Das $S$, Forer L, Schonherr S, Sidore C, Locke AE, Kwong A, Vrieze SI, Chew EY, Levy S, McGue M, et al. Next-generation genotype imputation service and methods. Nat Genet. 2016:48:1284-7.

23. McCarthy S, Das S, Kretzschmar W, Delaneau O, Wood AR, Teumer A, Kang HM, Fuchsberger C, Danecek P, Sharp K, et al. A reference panel of 64,976 haplotypes for genotype imputation. Nat Genet. 2016:48:1279-83.

24. Murray RP, Connett JE, Lauger GG, Voelker HT. Error in smoking measures: effects of intervention on relations of cotinine and carbon monoxide to self-reported smoking. The lung health study research group. Am J Public Health. 1993:83:1251-7.

25. Etter JF, Vu Duc T, Perneger TV. Saliva cotinine levels in smokers and nonsmokers. Am J Epidemiol. 2000;151:251-8.

26. Aulchenko YS, Ripke S, Isaacs A, van Duijn CM. GenABEL: an R library for genome-wide association analysis. Bioinformatics. 2007;23:1294-6.

27. Marchini J, Howie B, Myers S, McVean G, Donnelly P. A new multipoint method for genome-wide association studies by imputation of genotypes. Nat Genet. 2007:39:906-13.

28. Consortium" TaG. Genome-wide meta-analyses identify multiple loci associated with smoking behavior. Nat Genet. 2010:42:441-7.

29. Siedlinski M, Cho MH, Bakke P, Gulsvik A, Lomas DA, Anderson W, Kong X, Rennard SI, Beaty TH, Hokanson JE, et al. Genome-wide association study of smoking behaviours in patients with COPD. Thorax. 2011;66:894-902.

30. The UKKC. The UK10K project identifies rare variants in health and disease. Nature. 2015;526:82-90.

31. Wagner AH, Coffman AC, Ainscough BJ, Spies NC, Skidmore ZL, Campbell KM, Krysiak K, Pan D, McMichael JF, Eldred JM, et al. DGldb 2.0: mining clinically relevant drug-gene interactions. Nucleic Acids Res. 2016;44:D1036-44.

32. Wishart DS, Knox C, Guo AC, Shrivastava S, Hassanali M, Stothard P, Chang Z, Woolsey J. DrugBank: a comprehensive resource for in silico drug discovery and exploration. Nucleic Acids Res. 2006;34:D668-72.

33. Thorgeirsson TE, Gudbjartsson DF, Surakka I, Vink JM, Amin N, Geller F. Sequence variants at CHRNB3-CHRNA6 and CYP2A6 affect smoking behavior. Nat Genet. 2010;42:448-53.

34. Wain LV, Shrine N, Miller S, Jackson VE, Ntalla I, Artigas MS, Billington CK, Kheirallah AK, Allen R, Cook JP, et al. Novel insights into the genetics of smoking behaviour, lung function, and chronic obstructive pulmonary disease (UK BiLEVE): a genetic association study in UK biobank. Lancet Respir Med. 2015:3:769-81.

35. Lutz SM, Hokanson JE. Genetic influences on smoking and clinical disease. Understanding behavioral and biological pathways with mediation analysis. Ann Am Thorac Soc. 2014;11:1082-3.

36. Loukola A, Hällfors J, Korhonen T, Kaprio J. Genetics and smoking. Curr Addict Rep. 2014;1:75-82.

37. Hilberink SR, Jacobs JE, van Opstal S, van der Weijden T, Keegstra J, Kempers P, Muris JW, Grol RP, de Vries H. Validation of smoking cessation self-reported by patients with chronic obstructive pulmonary disease. Int J Gen Med. 2011:4:85-90

38. Benowitz NL, Hukkanen J, Jacob P. Nicotine chemistry, metabolism, kinetics and biomarkers. In: Henningfield J.E., London E.D., Pogun S. (eds) Nicotine Psychopharmacology. Handbook of Experimental Pharmacology, vol 192. Berlin, Heidelberg: Springer; 2009.

39. Patel YM, Stram DO, Wilkens LR, Park S-SL, Henderson BE, Marchand LL, Haiman CA, Murphy SE. The contribution of common genetic variation to nicotine and cotinine Glucuronidation in multiple ethnic/racial populations. Cancer Epidemiol Biomarkers Prev. 2015:24:119-27.

40. Benowitz NL. Nicotine addiction. N Engl J Med. 2010;362:2295-303. 
41. Zemen BG, Lai MH, Whitt JP, Khan Z, Zhao G, Meredith AL. Generation of Kcnmal (fl)-tdTomato, a conditional deletion of the BK channel a subunit in mouse. Physiol Rep. 2015;3:e12612.

42. Bell TJ, Miyashiro KY, Sul J-Y, McCullough R, Buckley PT, Jochems J, Meaney DF, Haydon P, Cantor C, Parsons TD, Eberwine J. Cytoplasmic BK(ca) channel intron-containing mRNAs contribute to the intrinsic excitability of hippocampal neurons. Proc Natl Acad Sci U S A. 2008;105:1901-6.

43. Buchhalter AR, Fant RV, Henningfield JE. Novel pharmacological approaches for treating tobacco dependence and withdrawal: current status. Drugs. 2008;68:1067-88.

44. Polston JE, Cunningham CS, Rodvelt KR, Miller DK. Lobeline augments and inhibits cocaine-induced hyperactivity in rats. Life Sci. 2006;79:981-90.

45. Liu YC, Lo YK, Wu SN. Stimulatory effects of chlorzoxazone, a centrally acting muscle relaxant, on large conductance calcium-activated potassium channels in pituitary GH3 cells. Brain Res. 2003:959:86-97.

46. Benowitz NL, Peng M, Jacob P 3rd. Effects of cigarette smoking and carbon monoxide on chlorzoxazone and caffeine metabolism. Clin Pharmacol Ther. 2003;74:468-74

47. Addolorato G, Leggio L, Hopf FW, Diana M, Bonci A. Novel therapeutic strategies for alcohol and drug addiction: focus on GABA, ion channels and transcranial magnetic stimulation. Neuropsychopharmacology. 2012;37:163-77.

48. Garcia-Arcos I, Geraghty P, Baumlin N, Campos M, Dabo AJ, Jundi B, Cummins N, Eden E, Grosche A, Salathe M, Foronjy R. Chronic electronic cigarette exposure in mice induces features of COPD in a nicotinedependent manner. Thorax. 2016;71:1119-29.

49. Bossé $\mathrm{Y}$, Postma DS, Sin DD, Lamontagne M, Couture C, Gaudreault N, Joubert $\mathrm{P}$, Wong $\mathrm{V}$, Elliott $\mathrm{M}$, van den Berge $\mathrm{M}$, et al. Molecular Signature of Smoking in Human Lung Tissues. Cancer Res. 2012;72:3753-63.

50. Cadet JL, Brannock C, Krasnova IN, Jayanthi S, Ladenheim B, McCoy MT, Walther D, Godino A, Pirooznia M, Lee RS. Genome-wide DNA hydroxymethylation identifies potassium channels in the nucleus accumbens as discriminators of methamphetamine addiction and abstinence. Mol Psychiatry. 2017;22:1196-204. 